\title{
The Helix Relation between Two Curves
}

\author{
ÍLKAY ARSLAN GÜVEN ${ }^{1, *}$, YUSUF YAYLI ${ }^{2}$ \\ ${ }^{1}$ Department of Mathematics, University of Gaziantep, Gaziantep, Turkey \\ ${ }^{2}$ Department of Mathematics, Ankara University, Ankara, Turkey \\ *Corresponding author: iarslan@gantep.edu.tr
}

Received August 14, 2013; Revised September 17, 2013; Accepted September 25, 2013

\begin{abstract}
In this study, we give the relation of being general helix and slant helix of two curves by using the equation between them. Also we find some results and express the characterizations of these curves.
\end{abstract}

Keywords: general helix, Frenet frame, Bertrand mates

Cite This Article: İLKAY ARSLAN GÜVEN, and YUSUF YAYLI, "The Helix Relation between Two Curves." Turkish Journal of Analysis and Number Theory 1, no. 1 (2013): 23-25. doi: 10.12691/tjant-1-1-6.

\section{Introduction}

The characterization of general helix was given with Lancret's theorem in 1802. Similarly, the general helix and the associated plane curve in the Euclidean 3-space $E^{3}$ were studied in [7]. It was clearly given that how to convert the associated plane curve to the general helix and vice versa. The equations of the general helix and associated plane curve were given to make this convertion. Also in [3], a similar equation was given to show that cylindrical helices can be constructed from plane curves.

In [2], the general helix and associated plane curve were studied in Minkowski 3-space $E_{1}^{3}$ finding new equations which showed how to obtain the general helix from the plane curve were given in $E_{1}^{3}$.

In this paper, we take the equation of two curves which is similar to the equation in [7]. Then we calculate the Frenet vectors and axis of symmetry of each curve and obtain the relation between them of how to be a general helix, slant helix and Bertrand mates.

\section{Preliminaries}

We now recall some basic notions about classical differential geometry of space curves in Euclidean space $E^{3}$.

Let $\beta: I \rightarrow \mathbb{R}^{3}$ be a curve with arc-length parameter $\mathrm{s}$ and let $\{T, N, B\}$ denote the Frenet frame of $\beta$. $T(s)=\beta^{\prime}(s)$ is called the unit tangent vector of $\beta$ at $s$. The curvature of $\beta$ is given by $\kappa(s)=\|\beta "(s)\|$. The unit principal normal vector $\mathrm{N}(\mathrm{s})$ of $\beta$ at $s$ is given by $\beta^{\prime \prime}(s)=\kappa(s) \cdot N(s)$. Also the unit vector $B(s)=T(s) \times N(s)$ is called the unit binormal vector of $\beta$ at $\mathrm{s}$. Then the famous Frenet formula holds

$$
\begin{aligned}
& T^{\prime}(s)=\kappa(s) \cdot N(s) \\
& N^{\prime}(s)=-\kappa(s) \cdot T(s)+\tau(s) B(s) \\
& B^{\prime}(s)=-\tau(s) N(s)
\end{aligned}
$$

where $\tau(s)$ is the torsion of $\beta$ at $s$.

Also the Frenet vectors of a curve $\alpha$, which is not given by arc-length parameter can be calculated as;

$$
\begin{aligned}
& T(t)=\frac{\alpha^{\prime}(t)}{\left\|\alpha^{\prime}(t)\right\|} \\
& B(t)=\frac{\alpha^{\prime}(t) \times \alpha^{\prime \prime}(t)}{\left\|\alpha^{\prime}(t) \times \alpha^{\prime \prime}(t)\right\|} \\
& N(t)=B(t) \times T(t)
\end{aligned}
$$

A curve $\beta: I \rightarrow \mathbb{R}^{3}$ is called a general helix if its tangent line forms a constant angle with a fixed straight line. This straight line is the axis of general helix. A classical result stated by Lancret says that "a curve is a general helix if and only if the ratio of the curvature to torsion is constant". If both curvature and torsion are nonzero constant, it is of course a general helix which is called circular helix.

A slant helix in $E^{3}$ is defined by the property that the principal normal line makes a constant angle with a fixed direction. In [4], it is shown that $\alpha$ is a slant helix in $E^{3}$ if and only if the geodesic curvature of the principal normal of the space curve $\alpha$ is a constant function.

Let two curves be $\alpha$ and $\beta$ in $E^{3}$. They are called Bertrand curves if their principal normal vectors are linearly dependent. We say that $\alpha$ and $\beta$ are Bertrand mates.

\section{The Equation of Two Curves}

Let $\bar{\alpha}$ be a curve and $\alpha$ be a unit speed general helix in $E^{3} . s$ denotes arc-length parameter of $\alpha$. The Frenet 
frame of $\bar{\alpha}$ and $\alpha$ are indicated by $\{\bar{T}, \bar{N}, \bar{B}\}$ and $\{T, N, B\}$, respectively. The curvatures of $\bar{\alpha}$ and $\alpha$ are $\bar{\kappa}$ and $\kappa$, the torsions are $\bar{\tau}$ and $\tau$. $a$ is the constant axis of general helix, $\theta$ is the angle between $a$ and $T$. The axis is given by

$$
a=\cos \theta T+\sin \theta B .
$$

The equation between $\bar{\alpha}$ and $\alpha$ is denoted in [7] as

$$
\bar{\alpha}=\alpha\left(s_{0}\right)+\sin \theta \cdot \alpha(s)+a .\left(s-s_{0}\right) \cos \theta
$$

where $\alpha\left(s_{0}\right)$ and $s_{0}$ are arbitrary constant vector and point.

Now, let find the Frenet vectors $\{\bar{T}, \bar{N}, \bar{B}\}$ of $\bar{\alpha}$. Since $\mathrm{s}$ is not arc-length parameter of $\bar{\alpha}$, we use the equations in (1).

The tangent vector $\bar{T}$ of $\bar{\alpha}$ is

$$
\bar{T}(s)=\frac{\sin \theta+\cos ^{2} \theta}{\sqrt{1+\cos \theta \cdot \sin 2 \theta}} T(s)+\frac{\cos \theta \cdot \sin \theta}{\sqrt{1+\cos \theta \cdot \sin \theta}} B(s),
$$

the principal normal vector $\bar{N}$ of $\bar{\alpha}$ is

$$
\bar{N}(s)=\left[\frac{\mu}{\sqrt{\lambda^{2}+\mu^{2}}} \cdot \frac{\sin \theta+\cos ^{2} \theta}{\sqrt{1+\cos \theta \cdot \sin 2 \theta}}-\frac{\lambda}{\sqrt{\lambda^{2}+\mu^{2}}} \cdot \frac{\cos \theta \cdot \sin \theta}{\sqrt{1+\cos \theta \cdot \sin 2 \theta}}\right] N(s)
$$

where

$$
\begin{gathered}
\lambda=\cos \theta \cdot \sin ^{2} \theta+\cos ^{3} \theta \cdot \sin \theta \\
\mu=\left(\sin \theta+\cos ^{2} \theta\right) \kappa-\left(\cos \theta \cdot \sin ^{2} \theta+\cos ^{3} \theta \cdot \sin \theta\right) \tau .
\end{gathered}
$$

Here if we say

$\frac{\mu}{\sqrt{\lambda^{2}+\mu^{2}}} \cdot \frac{\sin \theta+\cos ^{2} \theta}{\sqrt{1+\cos \theta \cdot \sin 2 \theta}}-\frac{\lambda}{\sqrt{\lambda^{2}+\mu^{2}}} \cdot \frac{\cos \theta \cdot \sin \theta}{\sqrt{1+\cos \theta \cdot \sin 2 \theta}}=c$

then we can denote the principal normal vectors as $\bar{N}(s)=c . N(s)$.

Also the binormal vector $\bar{B}$ of $\bar{\alpha}$ is found as

$$
\bar{B}(s)=\frac{\lambda}{\sqrt{\lambda^{2}+\mu^{2}}} T(s)+\frac{\mu}{\sqrt{\lambda^{2}+\mu^{2}}} B(s)
$$

We will state the following theorems whose proofs will be done by these calculations.

Theorem 1. Let a curve and a general helix be $\bar{\alpha}$ and $\alpha$, respectively. The equation between them is given by $\bar{\alpha}=\alpha\left(s_{0}\right)+\sin \theta \cdot \alpha(s)+a \cdot\left(s-s_{0}\right) \cos \theta$ where $a$ is the axis of general helix. If $\alpha$ is general helix then $\bar{\alpha}$ is a general helix.

Proof. Let the curve $\alpha$ be a general helix. The tangent and binormal vectors $T$ and $B$ of $\alpha$ make constant angle with a constant vector which can be a, the axis of $\alpha$.

$$
\begin{aligned}
& \langle a, T\rangle=\cos \theta \\
& \langle a, B\rangle=\sin \theta
\end{aligned}
$$

Since the tangent vector $\bar{T}$ of $\bar{\alpha}$ depends on $T$ and $B$, it also make constant angle with that constant vector.

$$
\langle a, \bar{T}\rangle=\frac{\cos \theta \cdot \sin \theta+\cos ^{3} \theta}{\sqrt{1+\cos \theta \cdot \sin 2 \theta}}+\frac{\cos \theta \cdot \sin ^{2} \theta}{\sqrt{1+\cos \theta \cdot \sin 2 \theta}}
$$

So $\bar{\alpha}$ is a general helix.

Theorem 2. Let a curve and a general helix be $\bar{\alpha}$ and $\alpha$, respectively. The equation between them is given by $\bar{\alpha}=\alpha\left(s_{0}\right)+\sin \theta \cdot \alpha(s)+a \cdot\left(s-s_{0}\right) \cos \theta$ where $\mathrm{a}$ is the axis of general helix. If the number $\mathrm{c}$ between the principal normal vectors of $\bar{\alpha}$ and $\alpha$ is constant, then $\alpha$ is slant helix if and only if $\bar{\alpha}$ is a slant helix.

Proof. The relation of principal normal vectors $\bar{N}$ and $N$ of $\bar{\alpha}$ and $\alpha$ is calculated as;

$\bar{N}(s)=\left[\frac{\mu}{\sqrt{\lambda^{2}+\mu^{2}}} \cdot \frac{\sin \theta+\cos ^{2} \theta}{\sqrt{1+\cos \theta \cdot \sin 2 \theta}}-\frac{\lambda}{\sqrt{\lambda^{2}+\mu^{2}}} \cdot \frac{\cos \theta \cdot \sin \theta}{\sqrt{1+\cos \theta \cdot \sin 2 \theta}}\right] N(s)$

which we denoted by $\bar{N}(s)=c \cdot N(s)$.

Here if $c$ is constant then $\bar{N}$ and $N$ are linearly dependent.

Firstly let $\alpha$ be a slant helix. Then the principal normal vector of $\alpha$ makes a constant angle with a fixed direction. Since the principal normal vector $\bar{N}$ is linearly dependent with $N, \bar{N}$ also makes a constant angle with that fixed direction. So $\bar{\alpha}$ is a slant helix.

The opposite of the proposition can be proved by following the same procedure.

Remark 1. The number c between the principal normal vectors was taken constant in the theorem. This number $\mathrm{c}$ is constant under the condition of $\kappa$ and $\tau$ are constant. Thus $\alpha$ is a circular helix. If $\alpha$ is a circular helix, then $\alpha$ is slant helix if and only if $\bar{\alpha}$ is a slant helix.

Theorem 3. Let a curve and a general helix be $\bar{\alpha}$ and $\alpha$, respectively. The equation between them is given by $\bar{\alpha}=\alpha\left(s_{0}\right)+\sin \theta \cdot \alpha(s)+a \cdot\left(s-s_{0}\right) \cos \theta$ where a is the axis of general helix. If the number $\mathrm{c}$ between the principal normal vectors of $\bar{\alpha}$ and $\alpha$ is constant, then $\bar{\alpha}$ and $\alpha$ are Bertrand mates.

Proof. In the equation $\bar{N}(s)=c \cdot N(s)$, let $\mathrm{c}$ be a constant number, then the principal normal vectors of $\bar{\alpha}$ and $\alpha$ are linearly dependent. So $\bar{\alpha}$ and $\alpha$ are Bertrand curves.

Remark 2. When $c$ is taken as a constant, then $\alpha$ is a circular helix. If $\alpha$ is a circular helix, then $\bar{\alpha}$ and $\alpha$ are Bertrand mates.

Now we will give an example

Example: Let $\alpha(s)=\left(6 s, 3 s^{2}, s^{3}\right)$ be a general helix with the curvature and torsion;

$$
\kappa(s)=\frac{2}{3\left(s^{2}+2\right)}, \tau(s)=\frac{2}{3\left(s^{2}+2\right)}
$$


The axis of $\alpha$ is calculated by $a(s)=\cos \theta T(s)+\sin \theta B(s)$. Here the angle $\theta$, between $a$ and $T$ is

$$
\frac{\kappa(s)}{\tau(s)}=\tan \theta=1 \Rightarrow \theta=\frac{\pi}{4} .
$$

If the vectors $\{T, N, B\}$ are Frenet vectors of $\alpha$, then

$$
\begin{gathered}
T(s)=\left(\frac{2}{s^{2}+2}, \frac{2 s}{s^{2}+2}, \frac{s^{2}}{s^{2}+2}\right) \\
N(s)=\left(\frac{-2 s^{3}-4 s}{\left(s^{2}+2\right)^{2}}, \frac{s^{4}-4 s^{2}-8}{\left(s^{2}+2\right)^{2}}, \frac{2 s^{3}+4 s}{\left(s^{2}+2\right)^{2}}\right) \\
B(s)=\left(\frac{s^{2}}{s^{2}+2}, \frac{-2 s}{s^{2}+2}, \frac{2}{s^{2}+2}\right)
\end{gathered}
$$

So the axis $a(s)$ is found as

$$
a(s)=\left(\frac{2 \sqrt{2}+\sqrt{2} s^{1}}{s^{2}+2}, 0, \frac{2 \sqrt{2}+\sqrt{2} s^{1}}{s^{2}+2}\right)
$$

The curve $\bar{\alpha}(s)$ is

$\bar{\alpha}(s)=\left(\frac{(3 \sqrt{2}+1) s^{3}+(6 \sqrt{2}+2) s}{s^{2}+2}, \frac{3 \sqrt{2}}{2} s^{2}, \frac{\frac{\sqrt{2}}{2} s^{5}+(\sqrt{2}+1) s^{3}+2 s}{s^{2}+2}\right)$

by taking the arbitrary constant vector $\alpha\left(s_{0}\right)=\overrightarrow{0}$ and arbitrary point $s_{0}=0$.
Then the Frenet vectors of $\bar{\alpha}$ are calculated by using $T$, $N, B$

$$
\begin{gathered}
\bar{T}(s)=\frac{1}{\sqrt{4+2 \sqrt{2}}}\left(1+\frac{2 \sqrt{2}}{s^{2}+2}, \frac{2 \sqrt{2} s}{s^{2}+2}, 1+\frac{\sqrt{2} s^{2}}{s^{2}+2}\right) \\
\bar{N}(s)=\frac{4+2 \sqrt{2}-3\left(s^{2}+2\right)^{2}}{\sqrt{4+2 \sqrt{2}} \cdot \sqrt{9\left(s^{2}+2\right)^{4}+8}} \cdot\left(\frac{-2 s^{3}-4 s}{\left(s^{2}+2\right)^{2}}, \frac{s^{4}-4 s^{2}-8}{\left(s^{2}+2\right)^{2}}, \frac{2 s^{3}+4 s}{\left(s^{2}+2\right)^{2}}\right) \\
\bar{B}(s)=\frac{1}{\sqrt{9\left(s^{2}+2\right)^{4}+8}}\left\{6\left(s^{2}+2\right)+\frac{2 \sqrt{2} s^{2}}{s^{2}+2},\right. \\
\left.6 s\left(s^{2}+2\right)-\frac{4 \sqrt{2} s}{s^{2}+2}, 3 s^{2}\left(s^{2}+2\right)-\frac{4 \sqrt{2}}{s^{2}+2}\right\}
\end{gathered}
$$

\section{References}

[1] M. Barros, General helices and a theorem of Lancret, Proceedings of the American Mathematical Society, 125-5 (1997),1503-1509.

[2] I.A. Güven, S. Kaya, Y. Yayli, General helix and associated plane curve in Minkowski 3-space, Far East Journal of Math. Sciences, 47-2 (2010), 225-233.

[3] S. Izumiya and N. Takeuchi, Generic properties of helices and Bertrand curves, Journal of Geometry, 74 (2002), 97-109.

[4] L. Kula and Y. Yayli, On slant helix and its spherical indicatrix, Applied Mathematics and Computation, 169 (2005), 600-607.

[5] W. Kühnel, Differential Geometry, second ed., Am. Math. Soc., 2006.

[6] D.J. Struik, Lectures on Classical Differential Geometry, Dover, New York, 1988.

[7] S. Sy, General helices and other topics in the differential geometry of curves, Master of Sci. in Mathematics, Michigan Technological Uni., 2001. 